Nicole Frerichs, DO

Department of Internal Medicine

and Geriatrics, Cleveland Clinic
Andrei Brateanu, MD, FACP

Department of Internal Medicine and Geriatrics,

Cleveland Clinic; Assistant Professor, Cleveland

Clinic Lerner College of Medicine of Case Western

Reserve University, Cleveland, $\mathrm{OH}$

\title{
Rhinosinusitis
}

\section{and the role of imaging}

\section{ABSTRACT}

Acute, uncomplicated rhinosinusitis is a clinical diagnosis. Imaging should only be used in the case of complicated sinus infections, recurrent or chronic sinus disease, or in surgical planning. The authors discuss key features of complicated and uncomplicated rhinosinusitis, management, and recommendations on the use of imaging in diagnosis.

\section{KEY POINTS}

When not clinically indicated, imaging increases healthcare costs and may expose patients to radiation and intravenous contrast unnecessarily.

Imaging in the setting of acute uncomplicated rhinosinusitis has not been shown to change clinical outcomes.

Computed tomography without contrast enhancement is the gold standard for sinus imaging when complications of rhinosinusitis are suspected. 3I-YeAR-OLD woman presents to the out-
patient clinic for evaluation of 2 weeks of nasal congestion and drainage, headache, and facial pressure. Her symptoms were mild at onset and seemed to improve over a few days, but then again worsened, and she has developed purulent nasal discharge. She is a smoker.

On clinical examination, she is afebrile, with mucosal edema and turbinate hypertrophy seen on anterior rhinoscopy. Based on her clinical presentation, she is suspected to have acute bacterial rhinosinusitis. Is imaging necessary to confirm this diagnosis?

\section{RHINOSINUSITIS: AN OVERVIEW}

Rhinosinusitis, the symptomatic inflammation of the nasal cavity and sinuses, ${ }^{1}$ can be divided into rhinitis and sinusitis, yet the two terms are often combined because the nasal mucosa and sinus mucosa are often inflamed synchronously. ${ }^{2}$ It is one of the most commonly treated conditions in ambulatory care, but the presentation is often similar to that of other upper respiratory tract infections, and accurate diagnosis is difficult. ${ }^{3}$ Symptoms commonly include nasal drainage, nasal obstruction, and facial pain or pressure. Other symptoms can include fever, headache, cough, ear pain or pressure, and anosmia. ${ }^{4}$

The diagnosis is generally based on symptoms and their duration. ${ }^{1}$ Acute rhinosinusitis is defined as up to 4 weeks of purulent nasal drainage accompanied by "nasal obstruction, facial pain-pressure-fullness, or both." In most cases, symptoms resolve in 7 to 10 days. Rhinosinusitis is subacute if symptoms persist beyond 4 weeks and less than 12 weeks, and chronic when symptoms last more than 12 weeks with objective evidence of mucosal inflammation 


\section{TABLE 1}

\section{Rhinosinusitis: Types and features}

\begin{tabular}{|c|c|c|c|}
\hline & Clinical features & Acute vs chronic & Complications \\
\hline Viral & $\begin{array}{l}\text { Symptoms improve } \\
\text { Duration }<10 \text { days }\end{array}$ & & \\
\hline Bacterial & $\begin{array}{l}\text { Symptoms persist > } 10 \text { days } \\
\text { "Double sickening": symptoms } \\
\text { improve, then worsen } \\
\text { High fever, then worsening symptoms }\end{array}$ & $\begin{array}{l}\text { Acute: }<4 \text { weeks } \\
\text { Subacute: } 4-12 \text { weeks } \\
\text { Chronic: }>12 \text { weeks } \\
\text { Recurrent: } 4 \text { or more episodes }\end{array}$ & $\begin{array}{l}\text { Uncomplicated: contained in nasal } \\
\text { cavity and sinuses } \\
\text { Complicated: spread to orbit, nervous } \\
\text { system, surrounding structures }\end{array}$ \\
\hline Fungal & $\begin{array}{l}\text { Seen in immunosuppression, chronic } \\
\text { steroid use, diabetes mellitus }\end{array}$ & $\begin{array}{l}\text { Acute: }<4 \text { weeks } \\
\text { Chronic: }>4 \text { weeks }\end{array}$ & $\begin{array}{l}\text { Noninvasive: contained within } \\
\text { sinuses } \\
\text { Invasive: spread beyond sinuses }\end{array}$ \\
\hline
\end{tabular}

Allergic Predominance of sneezing, rhinorrhea,

Chronic rhinosinusitis

nasal congestion and itching

\section{Rhinosinusitis is generally a clinical diagnosis}

visualized endoscopically or radiographically. Chronic rhinosinusitis may present with or without nasal polyps. ${ }^{1}$

Recurrent rhinosinusitis is defined as 4 or more episodes of acute rhinosinusitis per year with no symptoms between episodes. ${ }^{1}$

\section{INFECTIOUS VS NONINFECTIOUS}

Rhinosinusitis can be infectious or noninfectious. Infectious rhinosinusitis is classified as viral, bacterial, or fungal (Table 1).

\section{Viral rhinosinusitis}

Viral infection is the most common cause of rhinosinusitis and is diagnosed clinically when symptoms are present for less than 10 days and do not worsen. ${ }^{3}$

\section{Bacterial rhinosinusitis}

Bacterial infections are estimated to account for only $0.5 \%$ to $2 \%$ of rhinosinusitis cases. The gold standard for the diagnosis of bacterial sinusitis is a bacterial culture of the paranasal sinus cavity obtained by direct sinus aspiration..$^{5}$

Bacterial infection is suspected when symptoms are present for longer than 10 days without signs of clinical improvement, follow a biphasic pattern and initially improve but worsen after 5 to 6 days (referred to as "double sickening"), or are severe and include fever with temperature higher than $39^{\circ} \mathrm{C}\left(102^{\circ} \mathrm{F}\right),{ }^{3}$ purulent nasal discharge, or facial pain lasting more than 3 to 4 days. ${ }^{5,6}$

No single symptom is diagnostic of bacterial rhinosinusitis. It is estimated that purulent nasal secretions carry a sensitivity of 0.77 and a specificity of 0.54 , double sickening a sensitivity of 0.74 and specificity of 0.41 , and nasal congestion or obstruction a sensitivity of 0.83 and specificity of $0.24 .{ }^{7}$ In patients with all 3 symptoms of nasal discharge, nasal obstruction, and facial pain persisting longer than 10 days, only $40 \%$ to $50 \%$ have true bacterial sinusitis. $^{8}$

C-reactive protein testing has been used in addition to signs and symptoms to increase the accuracy of predicting acute bacterial sinusitis, but this has yet to be prospectively validated. $^{?}$

\section{Fungal rhinosinusitis}

Fungal rhinosinusitis refers to a wide variety of conditions that can present acutely in severely immunocompromised patients, or chronically in patients with mild immunosuppressive states such as diabetes mellitus or chronic corticosteroid use. ${ }^{9}$ It is categorized as acute (less than 4 weeks) or chronic (greater than 4 weeks), and as noninvasive or invasive.

Noninvasive fungal infection includes fungal colonization, fungus ball, and allergic fungal rhinosinusitis. ${ }^{10}$ Invasive fungal infections spread beyond the sinuses to involve bone, organs, or other structures. ${ }^{11}$

Infection needs to be distinguished from 
fungal colonization, encountered in patients with anatomic abnormalities such as nasal polyps. ${ }^{9}$ Fungal infections are also thought to have a role in the development of chronic rhinosinusitis. ${ }^{10}$

\section{Noninfectious rhinosinusitis}

Allergic rhinitis - an immune-mediated inflammatory response of the nasal mucous membranes after inhalation of allergensmay be seasonal, perennial, or episodic based on the exposure pattern to the triggering allergen. ${ }^{12}$ It is distinguished from infectious rhinosinusitis by the presence and predominance of sneezing, rhinorrhea, nasal congestion, and nasal itching. It is estimated to cause $30 \%$ of cases of acute maxillary rhinosinusitis. ${ }^{2}$

In allergic rhinosinusitis, purulent nasal drainage is not typically present, and patients do not have facial pain or pressure..$^{13}$

Migraine headache may also be associated with symptoms of rhinosinusitis, including sinus pressure, sinus pain, nasal congestion, runny nose, watery eyes, and itchy nose. In one study, $88 \%$ of patients self-diagnosed or physician-diagnosed with sinus headaches also fulfilled the International Headache Society criteria for migraine with or without aura. ${ }^{14}$

\section{Complications of infectious rhinosinusitis}

When rhinosinusitis spreads beyond the nasal cavity and sinuses to involve the orbit, nervous system, or other surrounding structures, ${ }^{15}$ complications can include preseptal or orbital cellulitis, abscess formation, meningitis, cavernous sinus thrombosis, and osteomyelitis. Although complications are uncommon, occurring in only 1 in 1,000 cases, ${ }^{3}$ they can be life-threatening. ${ }^{16}$

Ocular involvement should be suspected when patients present with ocular pain, eyelid swelling, pain with eye movements, visual changes, or displacement of the globe. ${ }^{17}$

Signs of central nervous system involvement, such as meningitis or intracranial abscess, include altered mental status, headache, nausea, vomiting, and fever. ${ }^{15}$ Involvement of the cavernous sinus should be suspected when palsy of cranial nerve III (oculomotor), IV (trochlear), or VI (abducens) is noted. ${ }^{17}$

Patients who present with these symptoms should be promptly evaluated for complicated infections. ${ }^{15}$

\section{MANAGEMENT: GENERAL APPROACHES}

When patients present with symptoms of acute rhinosinusitis believed to be uncomplicated based on review of history, observation is recommended for a period of 7 to 10 days, ${ }^{1,5}$ with symptomatic treatment including analgesics, intranasal glucocorticoids, intranasal saline irrigation, decongestants, and antihistamines. ${ }^{1,3,5}$ Analgesics including acetaminophen and nonsteroidal anti-inflammatory drugs are useful for relief of pain and fever. ${ }^{3}$ Intranasal corticosteroids are useful for reducing inflammation of nasal mucosa, thereby facilitating sinus drainage. ${ }^{1,5}$

\section{Glucocorticoids}

A 2013 Cochrane review found that patients who received intranasal glucocorticoids were more likely to experience symptomatic improvement compared with placebo, and higher doses brought more symptom relief. ${ }^{18}$

\section{Nasal irrigation}

Nasal saline irrigation has been shown to improve mucociliary clearance, but evidence of effectiveness is limited. One randomized controlled trial found that daily use of hypertonic nasal saline irrigation decreased nasal symptoms, but another study found no difference when no symptomatic treatment was compared with a combination of nasal saline irrigation, topical decongestants, and intranasal steroids. ${ }^{1,3}$

\section{Decongestants}

Decongestants, including oral and topical forms, are also options for symptom relief in rhinosinusitis. However, oral decongestants are not recommended due to a lack of clinical trials that have studied their effectiveness in acute sinusitis. 3,5

Xylometazoline nasal spray, a topical decongestant, was shown in 2 small studies to be effective at reducing congestion of sinus and nasal mucosa on imaging studies. ${ }^{3}$

Decongestants should be used with caution and for no longer than 3 to 5 consecutive days to prevent the development of rebound congestion. ${ }^{1}$

\section{Antihistamines}

Antihistamines are not recommended for the treatment of acute rhinosinusitis, as there are

\section{Nasal saline irrigation can improve mucociliary clearance, but evidence of effectiveness is limited}


no studies to support their effectiveness, and they may worsen congestion by causing excessive dryness of nasal mucosa. ${ }^{1}$

\section{Antimicrobials}

Evidence for the use of antimicrobials in acute bacterial sinusitis is weak due to a lack of standardization in diagnosis and duration of symptoms. In addition, $65 \%$ of patients thought to have acute bacterial rhinosinusitis treated with placebo improve spontaneously. ${ }^{5}$

Prescribing antibiotics is appropriate in cases of persistent and worsening symptoms. The Infectious Diseases Society of America (IDSA) recommends starting with amoxicillin and clavulanate when the clinical diagnosis of acute bacterial rhinosinusitis is made, and then monitoring for signs of improvement or worsening for 48 to 72 hours after initiation of treatment..$^{5}$ In contrast, the American Academy of Otolaryngology-Head and Neck Surgery suggests either antibiotics or a 7-day observation period ("watchful waiting"), with initiation of antibiotics if symptoms worsen or fail to improve during that time. ${ }^{1}$

The addition of clavulanate is recommended to improve the coverage of beta

Even with findings such as air fluid levels, plain radiography cannot distinguish viral from bacterial infection and $s o$ is not recommended lactamase-producing Haemophilus influenzae and Moraxella catarrhalis., ${ }^{3,5}$ If patients initially treated with amoxicillin with clavulanate do not demonstrate improvement, it is recommended to change antibiotics to either high-dose amoxicillin plus clavulanate, doxycycline, a respiratory fluoroquinolone such as moxifloxacin or levofloxacin, or a dual treatment of clindamycin plus a third-generation oral cephalosporin. ${ }^{3,5}$

Symptomatic treatments may also be prescribed as adjuncts to antibiotic therapy. The IDSA recommends nasal saline irrigation and intranasal glucocorticoids for acute bacterial rhinosinusitis. Decongestants and antihistamines are not recommended. ${ }^{5}$

In certain cases, consultation with a specialist should be sought. Patients with immunocompromised states, obstructive anatomic defects, recurrent sinusitis, fungal sinusitis, or suspected neoplasm should be evaluated by a specialist. Patients with severe symptoms such as persistent fever with temperature greater than $39^{\circ} \mathrm{C}\left(102^{\circ} \mathrm{F}\right)$, altered mental status, suspected ocular complications such as orbital cellulitis or intraorbital abscess, cavernous sinus thrombosis, or suspected neurologic complications such as meningitis or intracranial abscess should be referred to a specialist. ${ }^{3}$ Otolaryngology referral is appropriate for patients who have persistent symptoms despite initial treatment, patients with recurrent or chronic sinusitis, or in patients in whom anatomic abnormalities are suspected. ${ }^{2}$ Referral to ophthalmology or neurology for suspected serious ocular or central nervous system involvement may be warranted.

\section{IMAGING OPTIONS}

Rhinosinusitis is a clinical diagnosis. Imaging is reserved for cases of complicated rhinosinusitis, recurrent sinusitis, chronic rhinosinusitis, and immunocompromised patients. ${ }^{4}$ Imaging findings do not always correlate with symptoms. It is estimated that $3 \%$ to $40 \%$ of asymptomatic patients may have sinus abnormalities on computed tomography (CT). Thus, imaging should corroborate the presenting signs and symptoms. ${ }^{19}$ Indications for imaging are based on the classification of rhinosinusitis ${ }^{19}$ (Figure 1).

\section{Plain radiography}

Plain radiography can detect mucosal thickening, air fluid levels, opacification of the sinuses, anatomic variants, and foreign bodies, ${ }^{3}$ but it has poor sensitivity and specificity for sinus disease and thus is not usually recommended. ${ }^{20}$

\section{Computed tomography}

CT of the sinuses has become the gold standard for sinus imaging in the case of complicated sinus disease because of improved visualization of sinus anatomy. ${ }^{4}$

Cone-beam CT, a technique that creates 3-dimensional images of bony and soft-tissue structures of the face, is used primarily in dental imaging to evaluate the structures of the face, nasal cavity, and sinuses. ${ }^{21}$ It may be useful in the assessment of odontogenic sinusitis and maxillary sinus involvement. ${ }^{19}$

\section{Magnetic resonance imaging}

Magnetic resonance imaging (MRI) with and without intravenous contrast may be used to evaluate sinus disease, but it is not often the first imaging test performed. 


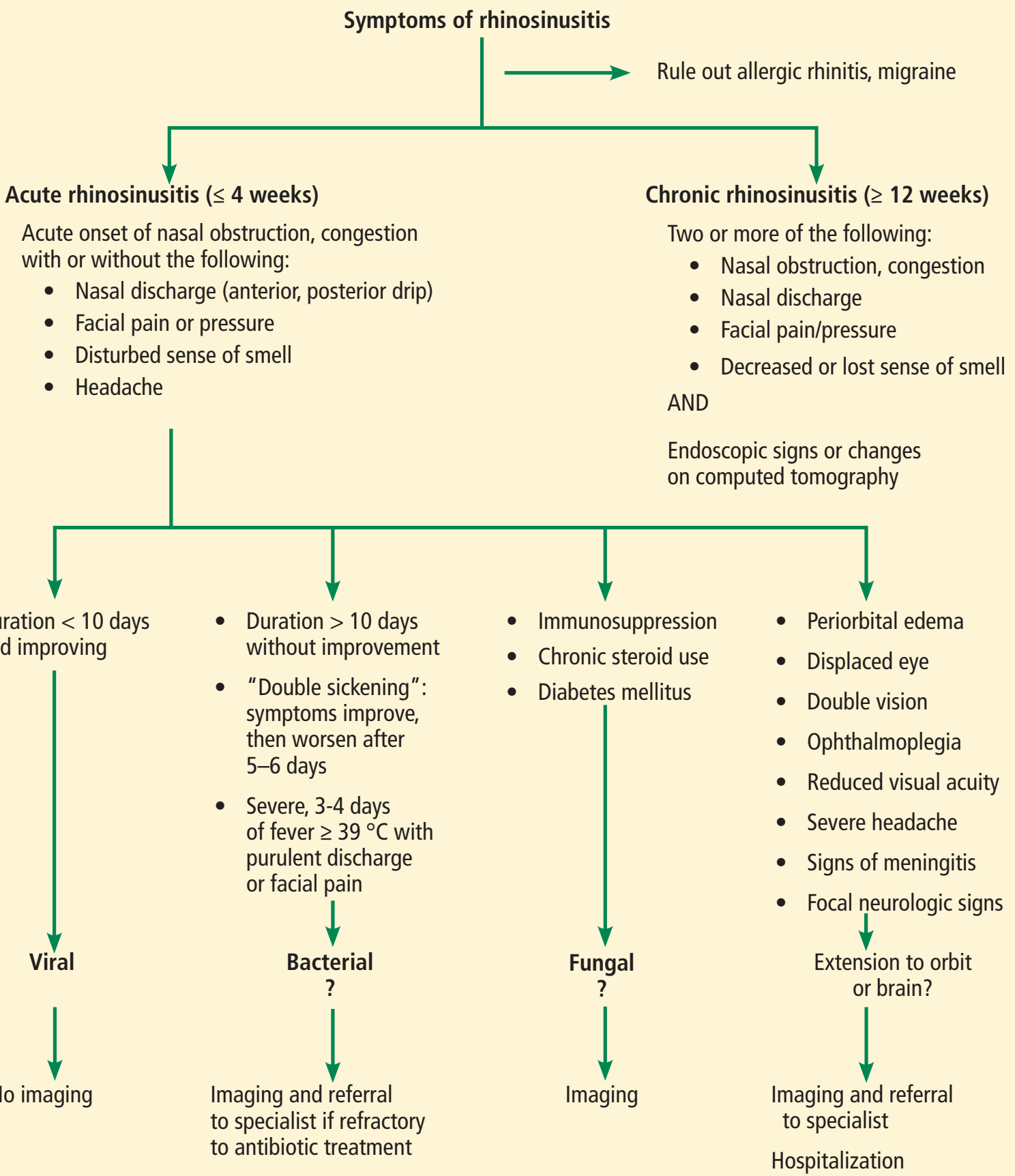

Figure 1. Approach to imaging in rhinosinusitis.

IMAGING IN ACUTE UNCOMPLICATED VIRAL OR BACTERIAL RHINOSINUSITIS

Referring back to our case of the 31-year-old woman, she presented with signs and symptoms consistent with acute, uncomplicated, likely bacterial rhinosinusitis, based on the duration of symptoms and double sickening. Acute, uncomplicated bacterial or viral rhinosinusitis is a clinical diagnosis, and patients who meet diagnostic criteria for uncomplicated rhinosinusitis should not undergo imaging. 4,19,22,23

Plain radiography in acute uncomplicated bacterial rhinosinusitis carries a sensitivity of 
$76 \%$ and a specificity of $79 \% .{ }^{20}$ But even in the presence of positive findings such as air fluid levels, plain radiography cannot distinguish between viral and bacterial infections and therefore is not recommended. ${ }^{3}$

In one study, CT of the nasal passages and sinuses performed in otherwise healthy patients who presented with "common cold" symptoms revealed a high prevalence of meatal and sinus findings, including occlusion of the ethmoid infundibulum in $77 \%$ of patients, abnormalities of one or both maxillary sinuses in $87 \%$, and ethmoid sinus abnormalities in $65 \%$ of patients. On repeat CT 2 weeks later, these findings had resolved or clearly improved in $79 \%$ of patients without antibiotic treatment. ${ }^{24}$ Thus, CT and MRI are not useful in the context of uncomplicated sinusitis. In addition, CT exposes patients to unnecessary radiation. ${ }^{4}$ When complications of rhinosinusitis or spread of infection are suspected, imaging can be considered. ${ }^{25}$

\section{IMAGING IN ACUTE COMPLICATED VIRAL OR BACTERIAL RHINOSINUSITIS}

In patients with suspected rhinosinusitis who

Findings on CT that suggest sinusitis include thickened mucosa, air fluid levels, and opacification of the sinuses present with symptoms indicating spread of infection beyond the sinuses and nasal cavity, imaging may be performed for diagnostic purposes. ${ }^{15}$

CT without contrast enhancement is the gold standard of sinus imaging and often the first test performed when complications of rhinosinusitis are suspected, as it affords the best delineation of bone and allows for visualization of bony integrity and erosion. Findings on CT suggestive of sinusitis include thickened mucosa (> $4 \mathrm{~mm}$ ), air fluid levels, and opacification of the sinuses. ${ }^{25}$

MRI may be indicated when complications such as aggressive intracranial or intraorbital spread of infection or cavernous sinus thrombosis is suspected, and for definition of soft-tissue masses. ${ }^{19}$ T1-weighted MRI is recommended to evaluate abscess or extension of infection past the sinuses, and T2-weighted MRI can differentiate inflammatory mucosa from soft-tissue masses. Contrast-enhanced MRI is recommended if cavernous sinus thrombosis is suspected.

When complications involving the orbit or cranium are suspected, the American College of Radiology recommends CT with contrast enhancement or MRI without contrast for evaluation. ${ }^{19}$ While the American College of Radiology notes that intravenous contrast is generally not needed, IDSA guidelines recommend contrast-enhanced CT with axial and coronal views in the case of suspected complications. ${ }^{5}$

\section{IMAGING IN RECURRENT ACUTE OR CHRONIC RHINOSINUSITIS}

Recurrent acute rhinosinusitis is defined as 4 or more episodes of acute rhinosinusitis per year, with no symptoms of rhinosinusitis between episodes. Chronic rhinosinusitis is symptom duration of more than 12 weeks with objective evidence of mucosal inflammation visualized endoscopically or radiographically. ${ }^{1}$ In either case, and in cases of sinonasal polyposis, imaging is warranted for evaluation and operative planning if surgery is warranted. ${ }^{19}$

Noncontrast CT is indicated as part of the workup before any surgical intervention, as it provides the best preoperative information, including delineation of complex anatomy, and may even be used intraoperatively to guide surgery. MRI is not first-line due to lack of bony detail. Cone-beam CT is useful in the assessment of odontogenic sinusitis and maxillary sinus involvement. ${ }^{19}$ Plain radiography may reveal foreign bodies or assist in diagnosing anatomic variants, but is not used clinically due to the superiority of CT.

In patients with a history of recurrent or chronic sinusitis who have had imaging in the past, in the absence of new symptoms, imaging does not provide further information and findings often remain unchanged. Repeat imaging is not necessary unless clinical signs or symptoms have changed. ${ }^{5}$

\section{IMAGING IN FUNGAL SINUSITIS}

Depending on the type of fungal infection suspected, imaging may be warranted. ${ }^{19}$ For saprophytic fungal infestations, which are frequently asymptomatic, the diagnosis is made clinically, and no imaging is required for diagnosis.

Fungus ball, another noninvasive fungal presentation, may be evaluated with CT of 
the sinuses or panoramic dental imaging; it appears as hyperattenuated material filling a single sinus.

Allergic fungal rhinosinusitis is the most common form of fungal sinus disease and is evaluated with CT or MRI. Classic findings on CT include the "double-density" sign caused by thick fungal mucin surrounded by hyperplastic mucosa. MRI with T1 and T2 weighting can be used to support the diagnosis.

When invasive acute or chronic fungal infection is suspected, contrast-enhanced CT or contrast-enhanced MRI can be used to visualize the sinuses, brain, and orbits. ${ }^{9}$ CT findings of invasive infection can include hypoattenuating mucosal thickening over the affected sinus and nasal cavity, bony erosion, and findings extending beyond the sinus and nasal cavities. MRI is time-consuming to obtain yet favorable for evaluating intracranial and intraorbital spread. ${ }^{10}$

\section{IMAGING IN ALLERGIC RHINITIS}

Imaging is not routinely recommended in patients who present with symptoms of allergic rhinitis. When patients present with symptoms of rhinosinusitis (as opposed to rhinitis,

\section{REFERENCES}

1. Rosenfeld RM, Piccirillo JF, Chandrasekhar SS, et al. Clinical practice guideline (update): adult sinusitis. Otolaryngol Head Neck Surg 2015; 152(2 suppl):S1S39. doi:10.1177/0194599815572097

2. Wilson JF. In the clinic. Acute sinusitis. Ann Intern Med 2010; 153(5):ITC31-15. doi:10.7326/0003-4819-153-5-201009070-01003

3. Aring AM, Chan MM. Current concepts in adult acute rhinosinusitis. Am Fam Physician 2016; 94(2):97-105. pmid:27419326

4. Kroll H, Hom J, Ahuja N, Smith CD, Wintermark M. R-SCAN: imaging for uncomplicated acute rhinosinusitis. J Am Coll Radiol 2017; 14(1):82-83.e1. doi:10.1016/j.jacr.2016.08.018

5. Chow AW, Benninger MS, Brook I, et al; Infectious Diseases Society of America. IDSA clinical practice guideline for acute bacterial rhinosinusitis in children and adults. Clin Infect Dis 2012; 54(8):e72-e112. doi:10.1093/cid/cir1043

6. Centers for Disease Control and Prevention (CDC). Antibiotic prescribing and use in doctor's offices. https://www.cdc.gov/antibiotic-use/community/for-hcp/ outpatient-hcp/adult-treatment-rec.html. Accessed June 5, 2020.

7. Ebell MH, McKay B, Dale A, Guilbault R, Ermias Y. Accuracy of signs and symptoms for the diagnosis of acute rhinosinusitis and acute bacterial rhinosinusitis. Ann Fam Med 2019; 17(2):164-172. doi:10.1370/afm.2354

8. Rubin MA, Ford LC, Gonzales R. Sore throat, earache, and upper respiratory symptoms. In: Kasper D, Fauci A, Hauser S, Longo D, Jameson JL, Loscalzo J. Harrison's Manual of Medicine. 20th ed. New York, NY: McGraw-Hill Education; 2019: Chapter 31.

9. Deutsch PG, Whittaker J, Prasad S. Invasive and non-invasive fungal rhinosinusitis-a review and update of the evidence. Medicina (Kaunas) 2019; 55(7). doi:10.3390/medicina55070319

10. Ponikau JU, Sherris DA, Kita H, Kern EB. Intranasal antifungal treatment in 5 patients with chronic rhinosinusitis. J Allergy Clin Immunol 2002; 110(6):862866. doi:10.1067/mai.2002.130051

11. Watkinson JC, Clarke RW. Scott-Brown's otorhinolaryngology and head and which affects only the nasal cavity), signs of complicated infections, signs of neoplasm, or persistence of symptoms and chronic rhinosiusitis, imaging may be warranted. As in the case of complicated rhinosinusitis, CT withut contrast is typically the first imaging test commended. ${ }^{12}$

\section{THE BOTTOM LINE}

Acute, uncomplicated rhinosinusitis remains clinical diagnosis. Imaging should only be sed in the case of complicated sinus infections, recurrent or chronic sinus disease, or in the case of surgical planning. ${ }^{25}$ Yet imaging is till frequently performed despite these recomendations. ${ }^{4}$

Imaging when not clinically indicated is associated with increased healthcare costs and nnecessary exposure to radiation and, in some cases, intravenous contrast material. ${ }^{4}$ Imaging in the setting of acute uncomplicated rhinosinusitis has not been proven to change clinical utcomes. ${ }^{26}$ Clinical judgment to carefully seect patients who are appropriate for imaging, s well as selecting low-dose radiation options when available, are ways to minimize imaging tilization.

neck surgery. 8th ed. Boca Raton, FL: CRC Press; 2018.

12. Seidman MD, Gurgel RK, Lin SY, et al; Guideline Otolaryngology Development Group. AAO-HNSF. Clinical practice guideline: allergic rhinitis. Otolaryngol Head Neck Surg 2015; 152(1 suppl):S1-S43. doi:10.1177/0194599814561600

13. Dykewicz MS, Hamilos DL. Rhinitis and sinusitis. J Allergy Clin Immunol 2010; 125(2 suppl 2):S103-S115. doi:10.1016/j.jaci.2009.12.989

14. Schreiber CP, Hutchinson S, Webster CJ, Ames M, Richardson MS, Powers C Prevalence of migraine in patients with a history of self-reported or physiciandiagnosed "sinus" headache. Arch Intern Med 2004; 164(16):1769-1772. doi:10.1001/archinte.164.16.1769

15. Ziegler A, Patadia M, Stankiewicz J. Neurological complications of acute and chronic sinusitis. Curr Neurol Neurosci Rep 2018; 18(2):5. doi:10.1007/s11910-018-0816-8

16. Wyler B, Mallon WK. Sinusitis update. Emerg Med Clin North Am 2019; 37(1):41-54. doi:10.1016/j.emc.2018.09.007

17. Younis RT, Lazar RH, Anand VK. Intracranial complications of sinusitis: a 15year review of 39 cases. Ear Nose Throat J 2002; 81(9):636-644. pmid:12353440

18. Zalmanovici Trestioreanu A, Yaphe J. Intranasal steroids for acute sinusitis. Cochrane Database Syst Rev 2013; (12):CD005149. doi:10.1002/14651858.CD005149.pub4

19. Expert Panel on Neurologic Imaging; Kirsch CF, Bykowski J, Aulino JM, et al. ACR Appropriateness Criteria Sinonasal Disease. J Am Coll Radiol 2017; 14(11S):S550-S559. doi:10.1016/j.jacr.2017.08.041

20. Lau J, Zucker D, Engels EA, et al. Diagnosis and treatment of acute bacterial rhinosinusitis: summary. AHRQ Evidence Report Summaries. https://www.ncbi. nlm.nih.gov/books/NBK11860/. Accessed June 5, 2020.

21. US Food and Drug Administration (FDA). Dental cone-beam computed tomography. https://www.fda.gov/radiation-emitting-products/medical-x-rayimaging/dental-cone-beam-computed-tomography. Accessed June 1, 2020.

22. Choosing Wisely. American Academy of Otolaryngology-Head and Neck Surgery Foundation. http://www.choosingwisely.org/clinician-lists/americanacademy-otolaryngology-head-and-neck-surgery-radiographic-imaging-for- 
uncomplicated-acute-rhinosinusitis/. Accessed June 1, 2020.

23. Slavin RG, Spector SL, Bernstein IL, et al; American Academy of Allergy, Asthma and Immunology; American College of Allergy, Asthma and Immunology; Joint Council of Allergy, Asthma and Immunology. The diagnosis and management of sinusitis: a practice parameter update. J Allergy Clin Immuno 2005; 116(6 suppl):S13-S47. doi:10.1016/j.jaci.2005.09.048

24. Gwaltney JM Jr, Phillips CD, Miller RD, Riker DK. Computed tomographic study of the common cold. N Engl J Med 1994; 330(1):25-30. doi:10.1056/ NEJM199401063300105

25. Eisenmenger LB, Anzai Y. Acute sinusitis in adults and children: evidence-based emergency imaging. In: Kelly A, Cronin P, Puig S, Applegate K, eds. Evidencebased Emergency Imaging: Optimizing Diagnostic Imaging of Patients in the Emergency Care Setting (Evidence-based Imaging). Springer; 2018.

26. Bhalla V, McCann A, Sykes K, Hoover L, Beahm DD, Chiu A. Assessing the clinical applicability of prior head imaging in patients with chronic rhinosinusitis. Int Forum Allergy Rhinol 2018; 8(1):20-24. doi:10.1002/alr.22042

Address: Andrei Brateanu, MD, FACP. Department of Internal Medicine, NA10, Cleveland Clinic, 9500 Euclid Avenue, Cleveland, OH 44195;

abratean@ccf.org 